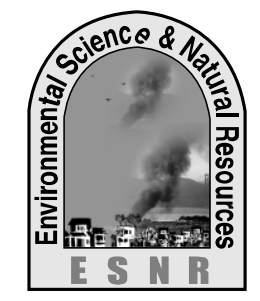

J. Environ. Sci. \& Natural Resources, 6(1): 11- 14, 2013

ISSN 1999-7361

\title{
Study on Contamination of Roadside Food by E. coli and Salmonella in Chittagong City Area
}

\author{
M. S. I. Khan ${ }^{1}$, M. R. Begum ${ }^{2}$, M. R. Haque ${ }^{3}$, M. A. Asgar ${ }^{4}$, M. S. Islam ${ }^{5}$ and A. S. M. G. \\ Kibria $^{6}$ \\ ${ }^{1}$ Dept. of Food Microbiology, ${ }^{3}$ Dept. of Biochemistry and Food Analysis, ${ }^{4}$ Dept. of Physiology \\ Biochemistry and Pharmacology, ${ }^{5}$ Dept. of Post-harvest Technology and Marketing, Patuakhali \\ Science and Technology University. \\ ${ }^{2}$ Dept. of Agricultural Economics and Social sciences, ${ }^{6}$ Dept. of Anatomy and Histology, \\ Chittagong Veterinary and Animal Sciences University.
}

\begin{abstract}
This study was conducted to determine the contamination of E. coli and Salmonella for different types of foods in Chittagong city area. In case of laboratory examination, almost half $(49.58 \%)$ of the samples were contaminated where $28.75 \%$ positive for E. coli. and $20.83 \%$ for Salmonella. The positive cases for type A sample was 20 out of 96 constituting $20.83 \%$ whereas the positive cases for type B sample was 49 out of 144 constituting $34.03 \%$ for E. coli. The positive cases of type A sample was 15 out of 96 making up $15.63 \%$ and for type B sample was 35 out of 144 making up 24.31\% for Salmonella.A chisquare $\left(\chi^{2}\right)$ test was used to examine the equality of observed proportions for $E$. coli and Salmonella of each item of both types of food where significant difference among the observed proportion for E. coli (p-value $<0.01)$ and Salmonella $(\mathrm{p}$-value $=0.032)$ for different items of A type and for E. coli (p-value<0.01) for different items of B type were observed and an odds ratio (OR) was measured for association between exposure and outcome where the probability of contaminated by E. coli of type B food is higher than contaminated of type A food ( $\mathrm{OR}=1.96$ and CI:1.07-3.58). The probability of contaminated by Salmonella of type B food was higher than type A food $(\mathrm{OR}=1.73$ and $\mathrm{CI}$ : 0.89-3.39). The comparatively high bacteria in type B samples indicated contamination from water, practice of inadequate hygienic measures, mishandling, improper storage, inadequate cooking and above all unhygienic condition of the retail shops.
\end{abstract}

Key Words: Contamination, E. coli, Roadside food, Salmonella.

\section{Introduction}

Food with proper nutritional value, hygienic in quality and appropriate in quantity is the topmost priority items for maintaining growth and development. Food of is essential for good health and active life (Potter, 1978). It was reported that, $90 \%$ of the population in Bangkok goes out a majority of the time for meals outside the homes. A total of $30 \%$ of the consumers was buying street food on a daily basis (Haque, 1999). Studies in developing countries have shown the potential for serious food poisoning out breaks due to microbial contamination and use of unpermitted food additives, food color, and presence of other adulterants (WHO, 1992). Street food survey in Bangkok, shows $84.4 \%$ food samples were contaminated and only $60 \%$ vendor washed utensils once a day (Haque, 1999). The transmission of human diseases through food, water and wastewater is a global problem, particularly of developing countries where gastrointestinal diseases are one of the most important causes of morbidity and mortality. However food habits adopted by populations may mitigate or increase the hazards (WHO, 1974 and 1976).

The quantitative study of bacterial contamination was done by Kawser (1997), Sami and Bari (1986), Zumra and Sami (1986) and Begum (1985). Kawser studied pour plate method to count the bacteria and Sami and Bari (1986), also used pour plate method but Begum (1985), determined total viable bacterial count using a modification of the surface drop technique.

Bangladesh being a developing country where sanitary practices of food shops are very poor, unhygienic and together with malnutrition, infections take a heavy toll of life every year. The people with different status usually produce and intake those food and get the chance of exposure of infection. Because only few vendor and consumer know about the hygienic condition of food. Although the study related to microbiological contamination of food is not so common in Bangladesh, but it is very important from the view point of public health as the significant number of city people used to take the road side food. Chittagong is the second largest city of Bangladesh with more than 4 million people. Past research indicates that study on contamination of roadside food of by E. coli and Salmonella in Chittagong district area was not done so far. Therefore the objective of this study was to conduct in different area of Chittagong city to assess the equality of observing proportion contamination of different foods by E. coli and Salmonella.

\section{Materials and Method}

The study was conducted in 17 different areas of Chittagong Metropolitan City from $15^{\text {th }}$ August 2009 to $10^{\text {th }}$ December 2009 under Department of Biochemistry and Food analysis, Faculty of 
Nutrition and Food Science, Patuakhali Science and Technology University. A total of 180 shops from where 240 food samples were collected. Microbiological examination of $E$. coli and Salmonella were done after dividing the shops into good quality shops (type A) and slum area shops (type B) on the basis of apparent hygienic condition. Good quality or type A food shops where interior decoration was beautiful and offering better quality foods, quick and amiable service of their employees and type B food shops were not so well decorated and they did not use tongs in serving.

\section{Collection of samples}

Twelve types of food samples, 8 from each items, were collected from good quality or type A food shops and 18 types of food samples, 8 from each items, were collected from slum area or mobile shops or type B food shops.
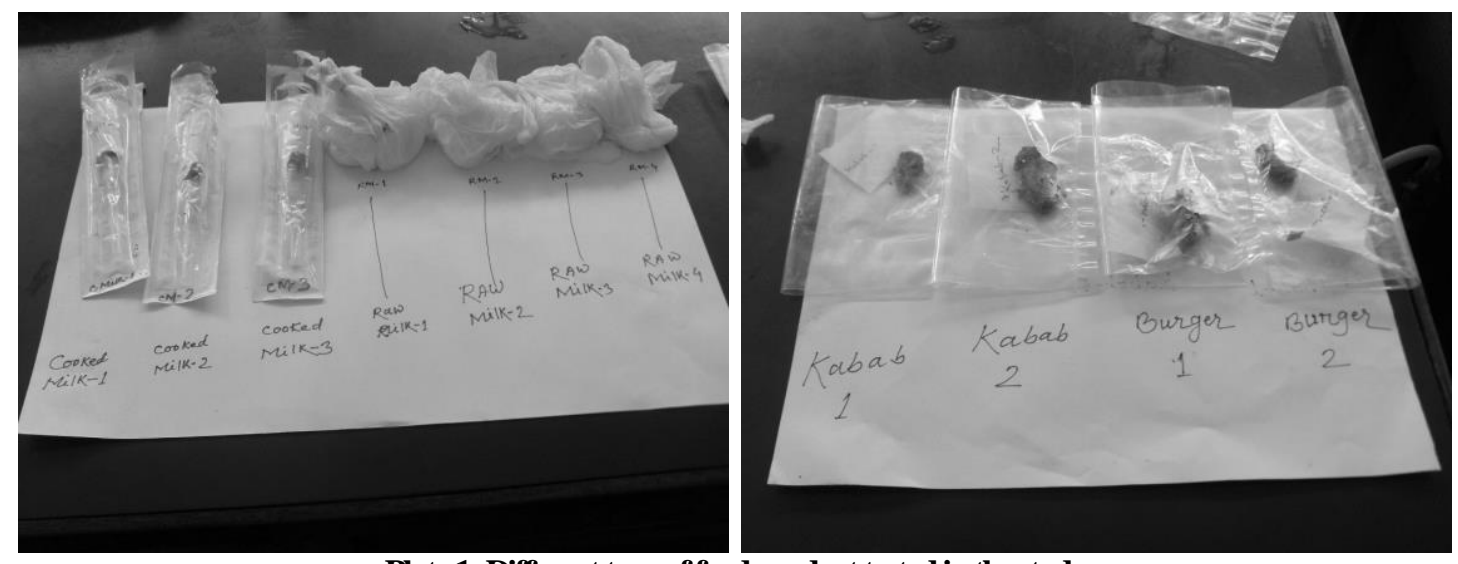

Plate 1: Different type of food product tested in the study

\section{Preparation of samples}

Homogenization of food samples was done by sterilized motor and pestle after taking $10 \mathrm{gm}$ of both superficial and inner samples. These meshed samples were inserted aseptically into sterile cotton plugged conical flask containing $0.9 \%$ sterile sodium chloride solution. After that these were mixed thoroughly by shaking for 20 times. The solution was allowed to stand for 5-10 minutes.

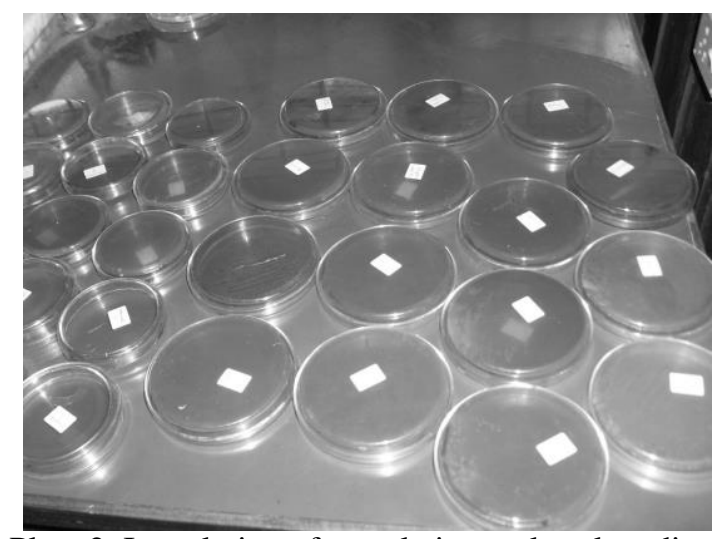

Plate 2: Inoculation of sample into cultural medium

\section{Preparation of Agar plate}

The petridishes were arranged and marked in a reasonable order for use. An aliquot was aseptically collected from the appropriate dilution and poured into the bottom of each petridish. After delivery the tip of the pipette was touched once to a dry spot in the dish. A separate sterile pipette was used to transfer an aliquot to each set of petridishes for each sample or sample dilution used. The undiluted samples and dilution containers were vigorously shaken before each transfer was done.

\section{Pouring Agar Plates}

Melted and cooled $\left(44-46^{\circ} \mathrm{c}\right)$ agar medium not less than $12 \mathrm{ml}$ (usually $12-15 \mathrm{ml}$ ) was added to each petridish containing an aliquot of the sample or its dilution. The inoculated medium 
was mixed carefully to prevent spilling. Care was taken not to splash the cover. The medium was allowed to set, then inverted and incubated for $18-48$ hours.

\section{Culture of $E$. coli}

MacConkey agar was used for culture of E. coli. For the preparation of the desired volume, the chemical ingredients of each media were first weighed and distilled water was added. The media was dissolved in water bath at boiling temperature. $\mathrm{p}^{\mathrm{H}}$ of the media was adjusted to 7.4-7.6 and then autoclaved at $121^{\circ} \mathrm{c}$ for 15 minutes at $15-\mathrm{lb} / \mathrm{sq}$. inch pressure. Inoculated plates were incubated for 24 hours at $37^{\circ} \mathrm{c}$ to facilitate E. coli growth.

\section{Culture of Salmonella}

Salmonella and Shigella agar (SS agar) were used for culture of Salmonella. For the desired volume, the chemical ingredients of each media were first weighed and distilled water was added. The media was dissolved in water bath at boiling temperature. $\mathrm{pH}$ of the media was adjusted to 7.4-7.6 and then autoclaved. Inoculated plates were incubated for 24 hours at $37^{\circ} \mathrm{c}$ to facilitate Salmonella growth.

\section{Data Analysis}

A chi-square test was applied to examine whether the observed proportion contaminated by E. coli and Salmonella are equal or not for each item for type A food and type B food respectively. An odds ratio was tested to examine the association between contaminated of type A and type B food by E. coli and Salmonella respectively.

\section{Results and Discussion}

Type A food consists of 12 items, for each item 8 samples were taken and tested whether the food was contaminated or not. A chi-square $\left(\chi^{2}\right)$ test was used to examine the equality of observed proportions for $E$. coli and Salmonella of each item. The result observed that there were significant difference among the observed proportion for $E$. coli (p-value $<0.01$ ) and Salmonella ( $\mathrm{p}$-value=0.032). Type B food consists of 18 items, for each item 8 samples were taken and tested whether the food was contaminated or not. A chi-square $\left(\chi^{2}\right)$ test was used to examine the equality of observed proportions for E. coli and Salmonella of each item. The result observed that there were significant difference among the observed proportion for $E$. coli (p-value<0.01) and no significant difference among the observed proportion for
Salmonella (p-value=0.066). This indicates contamination of the water by the street food vendors during its handling and storage. Similar results were reported from Peru and Colombia (FAO and PAHO, 1985).

An odds ratio (OR) is a measure of association between an exposure and an outcome. The odds ratio was conducted to examine the association between types of food and contaminated by $E$. coli and Salmonella respectively. The result was shown that the probability of contaminated by $E$. coli of type B food is higher than contaminated of type A food $(\mathrm{OR}=1.96$ and $\mathrm{CI}: 1.07-3.58)$. The probability of contaminated by Salmonella of type B food was higher than type A food (OR=1.73 and CI: 0.893.39). Overall the contamination of type A and B food by E. coli and salmonella were $28.75 \%$ and $20.83 \%$ respectively.Kawser (1997) reported that, of total 60 acceptable type A fast foods and drinks 11 $(15.07 \%)$ were completely sterile showing no microbial growth and rest $84.93 \%$ were contaminated. But in present study $49.58 \%$ of sample are contaminated and $50.52 \%$ are sterile. This low contamination of present study was due to this study include solely E. coli and Salmonella whereas Kawser (1997) study included all types of bacterial contamination.

\section{Conclusion}

The highest rate of contamination in different foods was found in street side shops and venders whereas in middle and high-class hotels the rate of contamination was low. This comparatively higher bacteria in type B samples suggests contamination from water, practice of inadequate hygienic measures, mishandling, improper storage, inadequate cooking and above all unhygienic condition of the retail shops.

\section{References}

Potter, N. N. 1978. Food Science, Third ed. CBS publishers \& Distributors, p-64.

Haque, M. Z. 1999. Socio-Economic and Environmental Aspects of Street Foods in Dhaka City, M. S. C. thesis, I.N.F.S.D.U., $p$ 8-14.

WHO, 1974. Expert committee microbiological aspects of food hygiene: WHO Tech Rep Ser. 598. 
WHO, 1976. Expert committee microbiological aspects of food hygiene: WHO Tech Rep Ser., 598:6, 8, 50-52.

WHO, 1992. Essential Safety Requirements for street Vended Foods. World Health Organization, Food safety unit.p12.

FAO and PAHO, 1985. Inform del Taller FAO/OPS Latinoamerica no Sobre Alimentos Vendidos en la via Publica, 21-25 October 1985,Lima, Peru. Pan American Health Organization, Washington, DC.

Kawser, M. 1997. Study of microbial aspect of different fastfoods and drinks to determine the fitness for human consumption. M.Sc thesis, I. N. F. S. D. U., p 1-10
Begum, M. 1985. Bacteriological analysis of Different foods to determine the fitness for human consumption. Journal of Pakistan Medical Association, 35:79.

Sami, Z. and Bari, A. 1986. Food Hygiene with reference to public health: Viable bacterial counts of ready to eat foods served in Rawalpindi, Islamabad. Journal of Pakistan medical association, 36:304-307.

Zumra and Sami, 1986. Food Hygiene with reference to public health. Micro biological contaminants of different foods in RawalPindi, Islamabad. J.P.M.A. June 36: 141-148. 